\title{
Walking barefoot vs. with minimalist footwear - influence on gait in younger and older adults
}

\author{
Evi Petersen ${ }^{1 *}$, Astrid Zech ${ }^{2}$ and Daniel Hamacher ${ }^{2}$
}

\begin{abstract}
Background: In recent years, minimalist footwear has been increasingly promoted for its use in sportive and recreational activities. These shoes are considered to function naturally like barefoot walking while providing a protective surface. Despite a growing popularity of these shoes in the older population, little is known about the influence of minimalist footwear on gait patterns. This study investigated whether overground walking with minimalist shoes is comparable to barefoot walking regarding gait stability and variability parameters.

Methods: In a randomized within-subject study design, 31 healthy younger ( $29 \pm 4$ years) and 33 healthy communitydwelling older adults (71 \pm 4 years) volunteered. Participants walked on flat ground, once barefoot and once with minimalist shoes. Gait variability of minimum toe clearance (MTC), stride length, stride time, and local dynamic gait stability were analysed.

Results: The results for both age groups showed significant condition effects (minimalist shoes vs. barefoot walking) for the outcomes of local dynamic stability $(p=.013)$, MTC variability $(p=.018)$, and stride length variability $(p<.001)$ indicating increased local dynamic stability and decreased gait variability during the minimalist shoe condition. Group effects (young vs. older adults) were detected in all gait outcomes.

Conclusion: Walking with minimalist shoes appeared to be associated with better gait performance than walking barefoot in both age groups. Thus, walking with minimalist shoes is not similar to barefoot walking. With respect to reducing the risk of falling, we suggest that minimalist shoes could be an alternative to barefoot walking or a transition option between shoes to barefoot for older adults.
\end{abstract}

Keywords: Elderly, Community-dwelling, Inertial sensors, Leguano, Minimalist shoes, Barefoot

\section{Background}

Bipedal gait is one of the most fundamental sensorimotor tasks performed every day [1]. Especially in older adults, a well-functioning gait pattern is recognized to be essential for autonomous participation in daily life [2]. With an increase in age, however, deficiencies in gait frequently evolve while the risk of falling increases [3].

\footnotetext{
* Correspondence: evi.petersen@usn.no

'Institute of Sports, Physical Education and Outdoor Life, University of South-Eastern Norway, Bø and Telemark, Norway

Full list of author information is available at the end of the article
}

Due to degenerative processes of the neuromuscular system and other age-related adaptations, older adults typically exhibit the following gait characteristics: (i) a wider stance and extended bipedal ground contact with shorter steps [4] as well as (ii) a diminished swing phase [5]. Besides internal factors, evidence reveals that footwear as an external criterion has a significant impact on the gait pattern [6, 7].

Footwear has been implicated as a factor in falls, which again is a crucial issue affecting health and quality of life in older adults $[8,9]$. In relation to this, older adults are

(C) The Author(s). 2020 Open Access This article is licensed under a Creative Commons Attribution 4.0 International License, which permits use, sharing, adaptation, distribution and reproduction in any medium or format, as long as you give appropriate credit to the original author(s) and the source, provide a link to the Creative Commons licence, and indicate if changes were made. The images or other third party material in this article are included in the article's Creative Commons licence, unless indicated otherwise in a credit line to the material. If material is not included in the article's Creative Commons licence and your intended use is not permitted by statutory regulation or exceeds the permitted use, you will need to obtain permission directly from the copyright holder. To view a copy of this licence, visit http://creativecommons.org/licenses/by/4.0/ The Creative Commons Public Domain Dedication waiver (http://creativecommons.org/publicdomain/zero/1.0/) applies to the data made available in this article, unless otherwise stated in a credit line to the data. 
often advised to wear shoes with low heels and firm slipresistant soles [10]. McKeon et al. [11] noted that permanent support to the foot might result in degenerative efficiency in foot muscles and sensitivity, and therefore carry a potential of adverse effects on the gait pattern. Accordingly, [11] suggest that walking barefoot is less restricting for motion control, which increases the sensitivity of the sensory mechanisms and activates the foot and lower leg muscles. Both sensory feedback sensitivity [12] and increased foot strength [13] showed to improve balance in older adults, and are therefore significant predictors in the prevention of falls. Thus, barefoot walking might result in beneficial effects on sensorimotor control.

In recent years, minimalist footwear (characterized by light weight, high flexibility and absence of cushioning material) has been increasingly promoted for its use in sportive and recreational activities. Wear of such footwear has been shown to be closely related to barefoot running conditions [14-16]. Therefore, it considered to function naturally like barefoot walking while providing a protective surface. Despite the growing popularity of these shoes in the older population, little is known about the influence of minimalist footwear on gait patterns and performance $[17,18]$. In relation to this, local dynamic gait stability, as well as gait variability measures, were associated with gait performance and the likelihood of falling $[19,20]$. More specifically, measures of local dynamic gait stability (LDS) are capable of distinguishing between cohorts of younger and older adults, while lower levels of LDS are associated with a higher risk of falling [21]. Furthermore, the minimum foot clearance (MFC) variability is a promising gait variable that can predict the risk of falling, with greater variabilities of MFC indicating a higher risk of falling [22].

In conclusion, walking with minimalist shoes seems to merge the positive effects of barefoot walking while providing a protective surface. However, the effects on the gait pattern were not sufficiently studied yet. Consequently, the primary aim of our study was to investigate if overground walking with minimalist shoes is comparable to barefoot walking regarding gait stability and variability. A study by Smith et al. [23] showed that minimalist shoes provided better overall and anterior-posterior static balance than walking barefoot. Additionally, [17] suggested that wearing minimalist shoes could provide benefits of barefoot walking in respect to fall prevention, while simultaneously offering some protection and support to the feet. Therefore, we predicted that overground walking with minimalist shoes is associated with higher gait stability and lower gait variability. We furthermore analyzed whether this relationship is similar in young and older adults.

\section{Methods \\ Participants}

In a randomized within-subject study design, gait data of 31 healthy younger (17 female, 14 male, age: $29 \pm 4$ years; BMI: $23 \pm 2$ ) and 33 healthy community-dwelling older (20 female, 13 male, age: $71 \pm 4$ years; BMI: $27 \pm 4$ ) participants were collected. We advertised in the local newspaper and at a local sport club to recruit the participants of this study.

Inclusion criteria were the age of $\leq 35$ years for the younger group and $\geq 65$ years for the older group. Participants had to be able to walk throughout 5 minutes without the need to pause or the use of assisting equipment. Additionally, participants had to be unfamiliar with regular barefoot walking or walking with minimalist shoes. Any self-reported motor-functional impairments that could affect gait performance, such as acute musculoskeletal disorders or neurological diseases, led to exclusion from the study. All subjects provided their written informed consent to their voluntary participation in this study as well as their allowance for publication, which has been approved by the local ethics committee (protocol no. FSV 16/13) and followed the principles of the Helsinki Declaration.

\section{Testing procedure}

To capture kinematic data, wireless inertial sensors (MTw2, Xsens Technologies B.V., Enschede, The Netherlands, range of measurement of angular velocity: \pm $1200 \mathrm{deg} / \mathrm{s}$, sampling rate: $100 \mathrm{~Hz}$ ) were attached to the participants' right forefeet using tape. The participants were block randomized to the different test condition sequences by using the computer-based program Research Randomizer (https://www.randomizer.org/). Further, participants were asked to walk at their preferred walking pace back and forth on a $25 \mathrm{~m}$ track inside a sports hall with flat ground. The following test conditions were performed in a randomized and balanced order: 1) barefoot walking and 2) walking with minimalist shoes (leguano classic, leguano $\mathrm{GmbH}$, Buchholz, Germany; material: $53 \%$ polyamide, $38 \%$ lyocell, $7 \%$ polypropylene, $2 \%$ elastane, Sole: $100 \%$ LIFOLIT $\left.^{\circ}\right)$. Participants walked for 3 minutes under each condition. The resting time between the test conditions was 5 minutes. Prior to each of the testing conditions, participants walked for approximately $1 \mathrm{mi}-$ nute back and forth the track to familiarize themselves with the corresponding test condition and to improve reliability [24].

\section{Data analysis and statistics}

The data analysis included gait variability measures as well as the local dynamic gait stability for which the largest Lyapunov exponent $(\lambda)$ was calculated. We removed the first strides (from the start to the first turn after 25 
m) of each trial as well as the last strides (from the last turn to the stop) to avoid possible transients, e.g. to analyze steady-state gait [25]. Furthermore, we removed the first $2.5 \mathrm{~m}$ of each section between two successive turns to exclude the acceleration periods after turning [26]. To account for possible deceleration periods before turning, the last $2.5 \mathrm{~m}$ were excluded from the subsequent analysis as well. At least 50 strides should be analyzed for gait variability measures [27]. In this study, the first 80 valid strides within each trial were analyzed.

To calculate the gait measures a) stride length, b) stride time and c) minimum toe clearance (MTC), we used a published algorithm [25]. As gait variability measures, the intraindividual standard deviations of each gait measure were analysed. To quantify $\lambda$, we timenormalized the three-dimensional angular velocity data of the 80 valid strides to 8000 samples. To reconstruct the state space, we applied the embedding approach. The time delay (9 samples) and the embedded dimension $(\mathrm{dE}=6)$ were determined, using the first minimum mutual information [28] and the global false nearest neighbors method, respectively [29]. The $\lambda$ was determined using the Rosensteins algorithm [30], which we implemented in MATLAB (version 2016a, TheMathWorks BV, Natrick, USA). The Euclidean distance of each nearest neighbor state space was tracked while the mean of the logarithm of the divergence curve was calculated. $\lambda$ is defined as the slope (linear fit) through $0-0.5$ strides. The implementation was validated previously [25]. Using IBM SPSS Statistics (V 21.0) for all statistical procedures, a two factor (condition: barefoot and minimalist footwear; age: younger and older adults) variance analysis with repeated measures was applied to identify differences across the two conditions.

\section{Results}

As Fig. 1 illustrates, the results show significant condition effects (minimalist shoes vs. barefoot walking) for the outcomes of local dynamic stability $(p=.013$, partial $\left.\eta^{2}=0.10\right)$, MTC variability $\left(p=.018\right.$, partial $\left.\eta^{2}=0.09\right)$, and stride length variability $\left(p<.001\right.$, partial $\left.\eta^{2}=0.26\right)$. These outcomes indicate increased local dynamic stability (lower largest Lyapunov exponent) and decreased gait variability during the minimalist shoe condition. There was no condition effect on stride time variability. Group effects (young vs. older adults) in all gait outcomes (LDS: $p<.001$, partial $\eta^{2}=0.30 ;$ MTC variability: $p=.006$, partial $\eta^{2}=0.12$; stride length variability: $p=.004$, partial $\eta^{2}=0.13$; stride time variability: $p=<.001$, partial $\left.\eta^{2}=0.26\right)$ were found. These effects indicate better LDS in younger participants and, surprisingly, better (lower) gait variability in older participants. There was no significant interaction effect between the walking condition and the group condition.
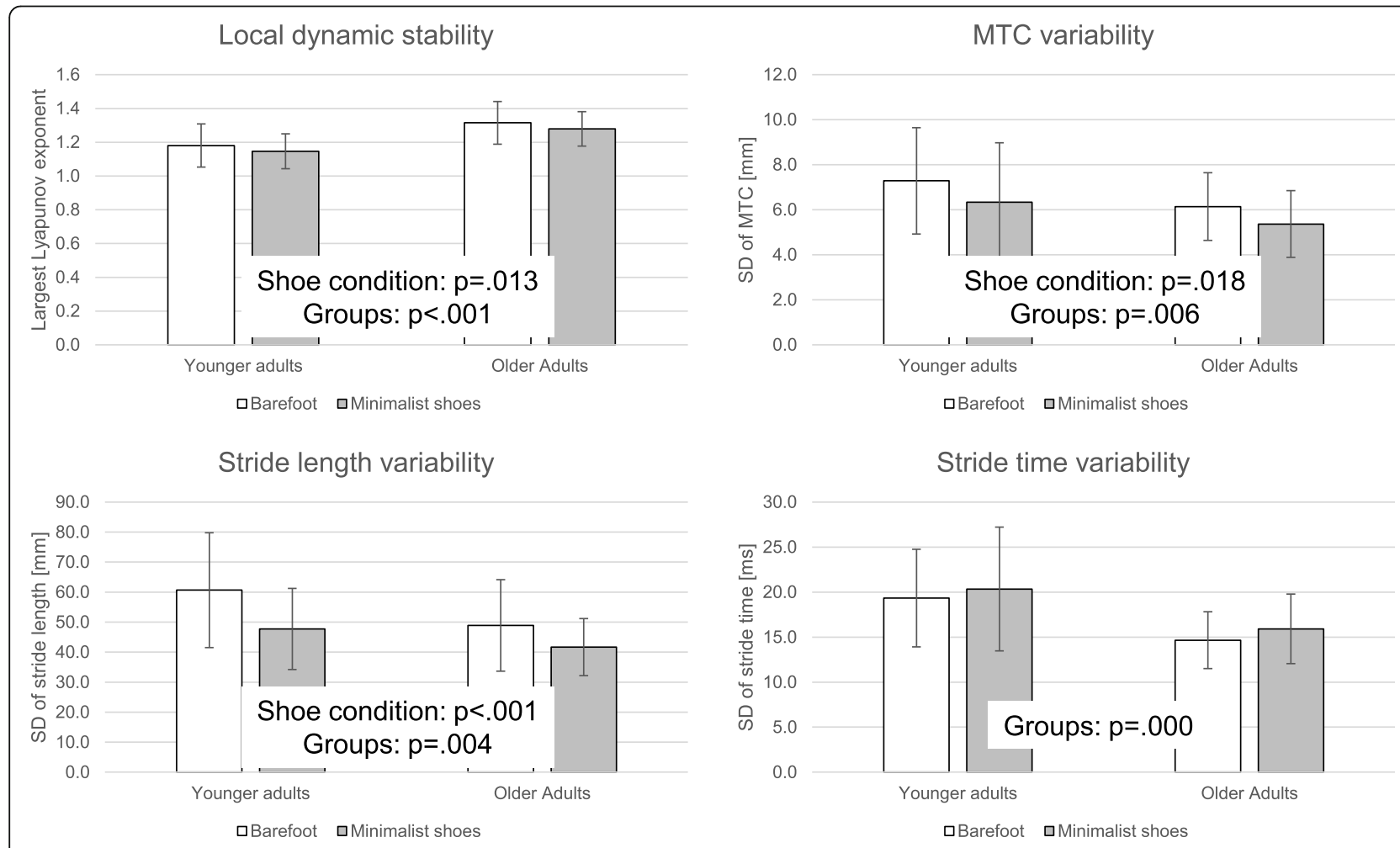

Fig. 1 Gait stability and variability in younger and older adults - barefoot vs. minimalist shoes 


\section{Discussion}

To the best of our knowledge, this is the first study that investigated whether overground walking with minimalist shoes is comparable to barefoot walking regarding gait stability and variability parameters in both younger and older adults. Current research discussed the use of minimalist shoes as an alternative to barefoot running or a method of transitioning between shod and barefoot running for athletes [23]. Overall and valid for both age groups, our results suggest that walking with minimalist shoes is associated with better gait stability and variability measures, thus, with a lower risk of falling [21, 22]. Consequently, walking with minimalist shoes is not similar to barefoot walking, and the positive effects of barefoot walking [11] are not necessarily generalizable to walking with minimalist shoes.

In order to reduce the risk of falling, we suggest that walking training with minimalist shoes could be an alternative to barefoot walking or a transition option between shoes to barefoot for older adults. Our proposition is in line with [17], who state that wearing minimalist shoes could provide benefits of barefoot walking in respect of fall prevention, while simultaneously offering some protection and support to the feet. Further, wearing minimalist shoes might help older adults to overcome different barriers, which are associated with walking barefoot. Often reported barriers are being ashamed of one's own feet, fear of falling or fear of instability as well as the sense of having cold feet [10,31].

Research on younger adults and children [15] showed that barefoot training can result in long-term outcomes, such as reduced ankle dorsiflexion at foot strike. That points to the prospect that wearing minimalist footwear regularly could have a positive impact on reducing the risk of falling. In one of their recent studies, Franklin et al. [32] investigated whether wearing minimalist shoes daily for 4 months could lead to improvements in balance and foot strength, which are both critical variables concerning a functional gait pattern. Preliminary results show that training with minimalist shoes can improve both parameters and thereby emphasize the potential of minimalist shoe use to reduce the risk of falling. That is in line with our results, which show that the use of minimalist shoes improved gait stability compared to barefoot walking.

We found less LDS in older adults compared to younger adults. This outcome has been frequently reported [19-21]. In contrast, it is surprising that older adults showed better (lower) gait variability than younger adults. A recent study by Hamacher et al. [33] reported similar outcomes. In their study, younger and older participants walked in their own shoes; however, the same system and algorithms were applied [33]. In the current study, younger adults show a much higher MTC, stride length and stride time variability during barefoot walking and walking with minimalist shoes compared to the younger participants of [33], walking in their own shoes. The older adults of the current study, on the other hand, show only a minor increase in gait variability compared to the older participants of the other study. We conclude that the group effect of the gait variability measures could be the result of younger adults adapting more quickly to an unfamiliar walking condition (walking barefoot / walking with minimalist shoes) to maintain local dynamic stability. This effect seems to be less pronounced in older adults. Since this is speculative, more research is needed to investigate the issue.

\section{Study limitations and future research implications}

We want to acknowledge four main study limitations. First, this research involved healthy community-dwelling older adults. Therefore, our current findings may not apply to older adults with health issues or already existing impairments. Most older adults have a higher risk of falling when confronted with existing dysfunctions concerning the gait pattern [34]. This fact points to the potential of future research within clinical settings.

Second, the participants of this study did not walk in their own shoes. Implementing this third condition to the testing procedure could have revealed more knowledge of how minimalist shoes influence gait in comparison to normal shoes, which are worn in daily life. Additionally, it would have made our results even more comparable to other studies. However, this question was not subject of our current study. We also ran our tests for only one type of minimalist shoe. Considering the growth of diversity in products, we encourage future research to compare the influence of different minimalist footwear as well as to include aspects of practicability regarding minimalist shoes for older adults.

Third, the application side in this study was a gym, proving a flat ground. Thus, our findings are limited to overground walking on an even surface. According to Zurales et al. [35], however, uneven surfaces are strong predictors of falls among older adults. Additionally and in line with Li et al. [36], falls occurred more often outdoors than indoors among older adults. Thus, future research should consider testing on an uneven surface, for instance, outdoors.

Last, this study focused on short-term effects. We suggest that future research should target long-term effects of fall prevention training with minimalist shoes or extended periods of wearing minimalist shoes during daily activity. Certainly, a systematic overview of the existing literature would enrich this scope of research.

\section{Conclusion}

This study demonstrates for walking in a straight line on flat ground that minimalist shoes, as compared to 
walking barefoot, have significantly different effects on gait and fall predicting parameters such as gait stability (LDS) and variability (MTC). Effects were observed for the group of younger as well as older adults. Walking with minimalist shoes was overall associated with better gait performance than walking barefoot. This finding hence demonstrates the potential of minimalist shoes as a means to prevent falls. We conclude that there is a need for future research to investigate the benefits of minimalist shoes for more complex walking tasks such as walking on uneven ground. Further, the long-term effects of minimalist usage need to be explored, particulary in relation to standard footwear. Simultaneously, we make a call for a systematic review of the literature concerned with minimalist footwear and the risk of falling.

\section{Abbreviations}

LDS: Local dynamic gait stability; MFC: Minimum foot clearance;

MTC: Minimum toe clearance; $\lambda$ : Lyapunov exponent

\section{Acknowledgments}

We thank all participants of this study and the two reviewer.

\section{Authors' contributions}

$E P, D H$ and $A Z$ were all fully involved in the planning of the study and preparation of the manuscript. EP collected the data for this study. EP and $\mathrm{DH}$ wrote the first draft of the manuscript and AZ advised. Each of the authors has read and concurs with the content in the final manuscript. The author(s) read and approved the final manuscript.

\section{Funding}

This research did not receive any grant from funding agencies in the public, commercial, or not-for-profit sectors.

\section{Availability of data and materials}

The dataset for this manuscript is not publicly available, however, requests to access the datasets can be directed to Evi Petersen (evi.petersen@usn.no).

\section{Ethics approval and consent to participate}

All subjects provided their written informed consent to their voluntary participation in this study and for the publication of their data. The study has been approved by the local ethics committee of Faculty of Social and Behavioural Sciences, Friedrich Schiller University Jena (protocol number: FSV 16/13) and followed the principles of the Helsinki Declaration.

\section{Consent for publication}

Not applicable.

\section{Competing interests}

None. The authors declare that the research was conducted in the absence of any commercial or financial relationships that could be construed as a potential conflict of interest.

\section{Author details}

'Institute of Sports, Physical Education and Outdoor Life, University of South-Eastern Norway, Bø and Telemark, Norway. ${ }^{2}$ Institute of Sports Science, Friedrich-Schiller University of Jena, Jena, Thuringia, Germany.

Received: 13 May 2019 Accepted: 20 February 2020

Published online: 04 March 2020

\section{References}

1. Bramble DM, Lieberman DE. Endurance running and the evolution of homo. Nature. 2004;432(7015):345-52.

2. Stolze $H$, Klebe $\mathrm{S}$, Zechlin C, Baecker C, Friege L, Deuschl G. Falls in frequent neurological diseases. J Neurol. 2004;251(1):79-84.
3. Maki BE. Gait changes in older adults: predictors of falls or indicators of fear? J Am Geriatr Soc. 1997;45(3):313-20.

4. Judge JO, Davis RB 3rd, Ounpuu S. Step length reductions in advanced age: the role of ankle and hip kinetics. J Gerontol A Biol Sci Med Sci. 1996;51(6):M303-12.

5. Jahn K, Zwergal A, Schniepp R. Gait disturbances in old age: classification, diagnosis, and treatment from a neurological perspective. Dtsch Arztebl Int. 2010;107(17):306-15 quiz 16

6. Lord SR. Shoe characteristics and balance in older women. J Am Geriatr Soc. 1996;44(4):429-33.

7. Connell BR, Wolf SL. Environmental and behavioral circumstances associated with falls at home among healthy elderly individuals. Arch Phys Med Rehabil. 1997;78(2):179-86.

8. Lord S, Sherrington C, Menz H, Close J. Falls in older people: risk factors and strategies for prevention. Cambridge: Cambridge University Press; 2007. https://doi.org/10.1017/CBO9780511722233.

9. Bloch F, Thibaud M, Tournoux-Facon C, Brèque C, Rigaud AS, Dugué B, et al. Estimation of the risk factors for falls in the elderly: can meta-analysis provide a valid answer? Geriatr Gerontol Int. 2013;13(2):250-63.

10. Menant JC, Steele JR, Menz HB, Munro BJ, Lord SR. Optimizing footwear for older people at risk of falls. J Rehabil Res Dev. 2008;45(8):1167-81.

11. McKeon PO, Hertel J, Bramble D, Davis I. The foot core system: a new paradigm for understanding intrinsic foot muscle function. Br I Sports Med. 2014:49:290.

12. Machado ÁS, da Silva CBP, da Rocha ES, Carpes FP. Effects of plantar foot sensitivity manipulation on postural control of young adult and elderly. Rev Bras Reumatol Engl Ed. 2017;57(1):30-6.

13. Spink MJ, Fotoohabadi MR, Wee E, Hill KD, Lord SR, Menz HB. Foot and ankle strength, range of motion, posture, and deformity are associated with balance and functional ability in older adults. Arch Phys Med Rehabil. 2011; 92(1):68-75

14. Perkins KP, Hanney WJ, Rothschild CE. The risks and benefits of running barefoot or in minimalist shoes: a systematic review. Sports Health. 2014; 6(6):475-80

15. Hollander K, Heidt C, Van Der Zwaard B, Braumann KM, Zech A. Long-term effects of habitual barefoot running and walking: a systematic review. Med Sci Sports Exerc. 2017;49(4):752-62.

16. Jenkins DW, Cauthon DJ. Barefoot running claims and controversies: a review of the literature. J Am Podiatr Med Assoc. 2011:101(3):231-46.

17. Franklin S, Grey MJ, Heneghan N, Bowen L, Li FX. Barefoot vs common footwear: a systematic review of the kinematic, kinetic and muscle activity differences during walking. Gait Posture. 2015;42(3):230-9.

18. Broscheid KC, Zech A. Influence of barefoot, minimalist, and standard footwear conditions on gait and balance in healthy older adults. J Am Geriatr Soc. 2016;64(2):435-7.

19. Bruijn $S$, Meijer $\mathrm{O}$, Beek P, Van Dieën J. Assessing the stability of human locomotion: a review of current measures. J R Soc Interface. 2013;10(83): 20120999.

20. Hamacher D, Singh N, Van Dieen J, Heller M, Taylor W. Kinematic measures for assessing gait stability in elderly individuals: a systematic review. J R Soc Interface. 2011;8(65):1682-98

21. Hamacher D, Hamacher D, Singh NB, Taylor WR, Schega L. Towards the assessment of local dynamic stability of level-grounded walking in an older population. Med Eng Phys. 2015;37(12):1152-5.

22. Barrett R, Mills P, Begg R. A systematic review of the effect of ageing and falls history on minimum foot clearance characteristics during level walking. Gait Posture. 2010;32(4):429-35.

23. Smith BS, Burton B, Johnson D, Kendrick S, Meyer E, Yuan W. Effects of wearing athletic shoes, five-toed shoes, and standing barefoot on balance performance in young adults. Int J Sports Phys Ther. 2015;10(1):69.

24. Hamacher D, Hamacher D, Krowicki M, Schega L. Between-day test-retest reliability of gait variability in older individuals improves with a familiarization trial. Aging Clin Exp Res. 2017;29(2):327-9.

25. Hamacher D, Hamacher D, Taylor WR, Singh NB, Schega L. Towards clinical application: repetitive sensor position re-calibration for improved reliability of gait parameters. Gait Posture. 2014;39(4):1146-8.

26. Lindemann U, Najafi B, Zijlstra W, Hauer K, Muche R, Becker C, et al. Distance to achieve steady state walking speed in frail elderly persons. Gait Posture. 2008;27(1):91-6.

27. König N, Singh NB, Von Beckerath J, Janke L, Taylor WR. Is gait variability reliable? An assessment of spatio-temporal parameters of gait variability during continuous overground walking. Gait Posture. 2014;39(1):615-7. 
28. Fraser AM, Swinney HL. Independent coordinates for strange attractors from mutual information. Phys Rev A Gen Phys. 1986;33(2):1134.

29. Kennel MB, Brown R, Abarbanel HD. Determining embedding dimension for phase-space reconstruction using a geometrical construction. Phys Rev A. 1992:45(6):3403.

30. Rosenstein MT, Collins JJ, De Luca CJ. A practical method for calculating largest Lyapunov exponents from small data sets. Physica D. 1993;65(1-2): $117-34$.

31. Mitty E. Nursing Care of the Aging Foot. Geriatr Nurs. 2009;30(5):350-4.

32. Franklin S, Grey MJ, Li F-X. Do minimalist shoes improve balance and foot strength in older adults? Footwear Sci. 2017;9(sup 1):S39-40.

33. Hamacher D, Hamacher D, Müller R, Schega L, Zech A. The effect of a cognitive dual task on the control of minimum toe clearance while walking. Mot Control. 2019;00:1-10.

34. Davis A, Haines T, Williams C. Do footwear styles cause falls or increase falls risk in healthy older adults? A systematic review. Footwear Sci. 2019;11(1): 13-23.

35. Zurales K, DeMott TK, Kim H, Allet L, Ashton-Miller JA, Richardson JK. Gait efficiency on an uneven surface is associated with falls and injury in older subjects with a spectrum of lower limb neuromuscular function: a prospective study. Am J Phys Med Rehabil. 2016;95(2):83.

36. Li W, Keegan THM, Sternfeld B, Sidney S, Charles P, Quesenberry J, Kelsey JL. Outdoor falls among middle-aged and older adults: a neglected public health problem. Am J Public Health. 2006;96(7):1192-200.

\section{Publisher's Note}

Springer Nature remains neutral with regard to jurisdictional claims in published maps and institutional affiliations.

Ready to submit your research? Choose BMC and benefit from:

- fast, convenient online submission

- thorough peer review by experienced researchers in your field

- rapid publication on acceptance

- support for research data, including large and complex data types

- gold Open Access which fosters wider collaboration and increased citations

- maximum visibility for your research: over $100 \mathrm{M}$ website views per year

At $\mathrm{BMC}$, research is always in progress.

Learn more biomedcentral.com/submissions 